\title{
Uma Abordagem para Identificação de Similaridade Entre Recursos Educacionais Utilizando Bases de Conhecimento Externas
}

\section{Title: An Approach to Identify Similarity Among Educational Resources Using External Knowledge Bases}

Laura Lima Dias

Pós-Graduação em Ciência da Computação

Universidade Federal de Juiz de Fora

Juiz de Fora - Minas Gerais - Brasil

laura_limadias@hotmail.com

\section{Eduardo Barrére}

Departamento de Ciência da Computação

Universidade Federal de Juiz de Fora

Juiz de Fora - Minas Gerais - Brasil

eduardo.barrere@ice.ufjf.br

\author{
Jayme Siqueira Barbosa \\ Bacharelado em Ciência da Computação \\ Universidade Federal de Juiz de Fora \\ Juiz de Fora - Minas Gerais - Brasil \\ jaymebarbosa@gmail.com \\ Jairo Francisco de Souza \\ Departamento de Ciência da Computação \\ Universidade Federal de Juiz de Fora \\ Juiz de Fora - Minas Gerais - Brasil \\ jairo.souza@ice.ufjf.br
}

\section{Resumo}

A necessidade de recuperar informações especificas e encontrar conteúdo complementar em grandes bases de dados tem se tornado notória dada a quantidade de mídias que são produzidas e armazenadas a cada instante. Nesse cenário, a educação tem se beneficiado da facilidade de produção de material didático em diversas mídias para criação de repositórios de recursos didáticos, o que gera, por outro lado, dificuldade na recuperação dessa informação tanto para aprendizes quanto para professores. Para ampliar as possibilidades do usuário encontrar recursos de interesse, este trabalho apresenta uma abordagem para identificar recursos similares em repositórios de recursos educacionais utilizando o sistema de categorização da DBpedia. Para validação, utilizou-se um benchmark criado a partir de videoaulas da Rede Nacional de Ensino e Pesquisa .

Palavras-Chave: similaridade, recuperação de informação, bases de conhecimento

\begin{abstract}
The need to retrieval specific information on videos and find complementary content in large databases has become notorious given the amount of media that are produced and stored at every moment. In this scenario, education also has encountered in the ease of production of novel instructional material a way to disseminate knowledge. On the other hand, it is not easy to retrieval such materials in large repositories. To expand the possibilities of the user to find resources of interest, this paper proposes an approach to identify similar resources in instructional resources repositories through the use of DBpedia categorizations. A benchmark composed by video lessons from Rede Nacional de Ensino e Pesquisa (Brazilian National Educational and Research Network) was used to evaluate the approach.
\end{abstract}

Keywords: similarity, information retrieval, knowledge bases

Cite as: Dias, L. L., Barbosa, J. S., Barrére, E. \& De Souza, J. F. (2017). An Approach to Identify Similarity Among Educational Resources Using External Knowledge Bases (Uma Abordagem para Identificação de Similaridade Entre Recursos Educacionais Utilizando Bases de Conhecimento Externas). Brazilian Journal of Computers in Education (Revista Brasileira de Informática na Educação - RBIE), 25(2), 18-37. DOI: $10.5753 /$ RBIE.2017.25.02.18 


\section{Introdução}

A facilidade de criação de conteúdos digitais tem permitido que repositórios de recursos educacionais possam crescer rapidamente, gerando necessidade de melhores mecanismos de classificação e busca desses conteúdos. De todas as mídias, o vídeo é a que tem o maior destaque quando o assunto é educação, seja pela sua capacidade de adaptação às diversas plataformas (Oliveira, Santana, \& Pontes, 2010) ou pela sua atratividade natural, unindo fatores auditivos e visuais numa única mídia (Figueiredo, Assireu, \& Souza, 2015; Medeiros \& Pansanato, 2015). Por outro lado, para usuários dos repositórios de videoaulas, é quase impossível encontrar vídeos de interesse quando se utiliza apenas pequenos trechos de texto associado ao vídeo, como título e alguns metadados disponíveis nos repositórios que quase sempre são curtos e de alto nível (Yang \& Meinel, 2014).

O fato dos usuários ainda não conseguirem extrair o máximo potencial em suas pesquisas, mostra a relevância desses estudos, principalmente quando estão relacionados a vídeos, onde a linguagem natural muitas vezes é vaga e incerta e o conteúdo dificilmente é bem representado semanticamente (Gupta, Saini, \& Saxena, 2015). Se tratando dos vídeos disponíveis na internet, isso se torna ainda mais evidente, pois existem grandes demandas para solucionar esse problema, o que atrai muito interesse entre os pesquisadores (Jiang, Bhattacharya, Chang, \& Shah, 2013).

Quando se pensa em educação, diversos vídeos estão disponibilizados em repositórios de objetos de aprendizagem. Apesar do fato desses repositórios serem bem estruturados, a inserção de informações relativas ao objeto de aprendizagem depende, na maioria absoluta das vezes, da ação direta de uma pessoa. Essa ação pode gerar um baixo uso dos repositórios de objetos de aprendizagem, pois nem sempre os termos corretos são cadastrados para que o objeto seja encontrado pelos usuários finais. Neste sentido, destaca-se a falta de conhecimento dos autores de vídeos educacionais (como exemplo significativo de objetos de aprendizagem) na definição dos termos que servirão para que os usuários encontrem os vídeos disponibilizados. Neste cenário, se faz necessária a geração de novos termos a serem utilizados nos mecanismos de busca (Diana, Nakayama, Spanhol, \& Silveira, 2015).

Uma solução para este problema é tentar melhorar a qualidade dos termos de busca a serem utilizados nos mecanismos de busca de conteúdos educacionais. Casagrande, Kozima, e Willrich (2015) apresentam essa solução para a recomendação de conteúdos em repositórios digitais, considerando a existência de metadados que descrevem os conteúdos e estão presentes em diversas partes do cadastro do objeto de aprendizagem, contribuindo assim para que o usuário final tenha maior êxito na busca por um determinado termo.

Outra solução é apresentada por Neves, Brandão, e Ishitani (2016), ao proporem uma metodologia que emprega ontologias, anotação automática de metadados, recuperação de informação e mineração de textos para recomendação automática e agregação de conteúdos relacionados, em conformidade com o padrão SCORM para objetos de aprendizagem. A solução apresentada no presente trabalho emprega parte dessas tecnologias, mas tem como foco principal a mídia vídeo em um cenário genérico, independente do vídeo estar ou não disponibilizado como um objeto de aprendizagem, pois quando se pensa em vídeos educacionais, eles também são disponibilizados por sites de grandes Universidades (MIT, Stanford, Princeton, Columbia, Unicamp etc.), em sites de empresas privadas (YouTube, iTunes etc.) e também nos sites de organizações sociais, como é o caso da Rede Nacional de Ensino e Pesquisa (RNP), que disponibiliza os serviços vídeo@RNP e videoaula@RNP. Nesses cenários os vídeos também podem ter enfoque no ambiente acadêmico ou ensino em geral, como relata (Barrére, 2014; Cunha \& Faustino, 2014; McGreal, 2004), apresentando diversas vezes conteúdos complementares (vídeos, apostilas etc.), 
o que evidencia a necessidade de uma estrutura de organização e classificação dos mesmos a fim de facilitar o processo de aprendizagem.

Vale também destacar que o sucesso em encontrar o vídeo relacionado ao tema que está sendo buscado muitas vezes pode proporcionar um aprendizado mais completo para o aluno. Porém, nem sempre o aluno tem amplo conhecimento sobre o tema e seus termos correlatos, e a interface de busca não lhe oferece o apoio necessário para que tal busca tenha resultados satisfatórios (Nash, 2005).

Uma alternativa para sanar essa necessidade é o desenvolvimento de um sistema de classificação, onde seja possível catalogar vídeos e outros recursos didáticos nos repositórios, de modo que os usuários possam recuperá-los (Nash, 2005). Outra alternativa é a criação de Sistemas de Recomendação que, segundo Da Motta e Lopes (2002), são baseados na premissa de que informações que foram encontradas e avaliadas anteriormente podem servir para as buscas que serão feitas por outras pessoas.

As recomendações são um dos métodos essenciais da Recuperação de Informação, principalmente quando se trata de descoberta de conteúdo nos ambientes atuais que são ricos em informação. Tudo isso combinado com pesquisa pura e navegação, permite que os usuários enfrentem esse grande volume de informação de uma forma mais satisfatória (Davidson et al., 2010). Shi, Larson, e Hanjalic (2014) afirma que, nas duas últimas décadas, tem sido dedicado um grande esforço na pesquisa e desenvolvimento de algoritmos que geram recomendações.

Para chegar a um repositório de objeto de aprendizagem de fácil navegação e bem classificado, ou a um sistema de recomendação satisfatório, existe um caminho a percorrer, pois, em ambos os casos, está implícito que já é conhecido o assunto tratado no conteúdo digital. $\mathrm{O}$ ato de explicitar certos aspectos dos documentos, principalmente em vídeos, a serem publicados, recebe o nome de anotação, ou seja, marcas colocadas nos documentos para instruir do que são compostos, com a finalidade de facilitar o entendimento e o assunto abordado nos mesmos (Ramalho, 2000).

Mídias anotadas facilitam o processo de busca em repositórios e muitos pesquisadores tem trabalhado em melhorias no processo de anotação para diversas mídias (Dasiopoulou, Giannakidou, Litos, Malasioti, \& Kompatsiaris, 2011; Qazi \& Goudar, 2016).

Anotações geralmente representam o tópico central da mídia e nem sempre são suficientes para resolver os interesses de busca dos usuários. Para permitir melhor uso dessas anotações, este trabalho apresenta uma abordagem para identificar conteúdos similares através do conhecimento existente em bases externas. Utilizamos neste trabalho a DBpedia como base de conhecimento e fazemos uso do grafo de informações contido na DBpedia para calcular a similaridade de recursos dentro do repositório de conteúdos educacionais. O conteúdo enciclopédico da DBpedia se mostra adequado para calcular similaridade em repositórios de conteúdos educacionais por possuir boa cobertura nos principais tópicos de ensino (Halavais \& Lackaff, 2008).

Para melhor apresentação do trabalho, o texto está organizado da seguinte forma: os trabalhos relacionados são apresentados na seção 2; na seção 3 são discutidos os métodos de anotação semântica presentes na literatura; na seção 4 são apresentadas as bases de conhecimentos que podem ser utilizadas para adicionar conhecimento de domínio neste tipo de abordagem; a seção 5 apresenta a formalização da abordagem proposta para a construção dos relacionamentos e, em seguida, são apresentadas a avaliação dessa abordagem. Na última seção encontram-se as conclusões e a discussão de trabalhos futuros. 


\section{Trabalhos Relacionados}

Com o expressivo volume de dados ligados que vem sendo publicado na web, em sistemas de recuperação de informação, já é efetivo seu uso. Dados ligados podem ser usados para agregar valor ao conjunto de dados original (Araújo \& De Souza, 2011), desenvolvendo-o com associação a outros conjuntos de dados conexos, além de ser possível utilizá-los de diferentes formas e para diferentes propósitos, como é o caso do Sindice (Oren et al., 2008). Além disso, essas bases externas de conhecimento tem sido utilizadas para apoiar tarefas, tais como anotação semântica (Mendes, Jakob, García-Silva, \& Bizer, 2011), alinhamento de entidades (Jain, Hitzler, Sheth, Verma, \& Yeh, 2010), identificação de contexto (Kawase, Siehndel, Pereira Nunes, Herder, \& Nejdl, 2014), dentre outras. Neste trabalho fazemos uso do sistema de categorias da DBpedia para determinar a similaridade entre recursos educacionais anotados semanticamente.

Conforme discutido em (Herrera, Casanova, Nunes, Lopes, \& Leme, 2016), à partir de uma entidade da DBpedia, é possível percorrer diversos caminhos distintos no grafo de relacionamentos para alcançar uma dada entidade. $\mathrm{O}$ grau de similaridade entre entidades pode ser medido de acordo com a quantidade de percursos entre duas entidades, a distância desses percursos e os tipos de relações existentes nestes percursos, como é o caso do DBpedia Profiler (Herrera et al., 2016) e dos trabalhos de Zhu e Iglesias (2017) e de Cheniki, Belkhir, Sam, e Messai (2016). Nestes três trabalhos, diferentes abordagens para encontrar caminhos entre entidades e determinar suas similaridades são propostas. Conforme discutido pelos autores, para um melhor desempenho o cálculo de similaridade necessita processar previamente toda a base da DBpedia, o que exige um reprocessamento a cada atualização da base de conhecimento. Por outro lado, ao fazer uso de todo o grafo da DBpedia, o valor de similaridade entre entidades possui boa acurácia. Assim, é possível analisar a similaridade entre recursos educacionais através da similaridade entre cada par de anotações.

Se os recursos educacionais tiverem outro tipo de informação associada, como texto, abordagens similares são encontradas na literatura. Por exemplo, o sistema de categorias pode ser utilizado para encontrar o tópico abordado pelo recurso educacional, como no trabalho de Hulpus, Hayes, Karnstedt, e Greene (2013) e Köohncke e Balke (2010). Ambos os trabalhos utilizam informação textual e as relaciona com entidades da DBpedia para, em seguida, processar o grafo de relações entre entidades e identificar o tópico que melhor representa um documento. Neste processo, ambos os trabalhos precisam tratar de problemas como identificação de recursos candidatos e desambiguação de recursos, visto que um mesmo termo no texto pode representar diferentes entidades.

No cenário tratado neste artigo, um recurso educacional pode ter várias anotações, as quais podem fazer parte de diversas categorias distintas. A similaridade é calculada através das categorias em comum nas quais essas anotações se ligam no grafo da base de conhecimento. Assim, quanto mais os recursos educacionais compartilharem de um mesmo assunto, mais similares eles serão. Como será visto na seção 6 , o uso desse subgrafo é suficiente para gerar bons resultados, o que acarreta uma quantidade menor de dados a serem processados e, por consequência, um melhor tempo de processamento.

Com a dificuldade de recuperar informação presentes em vídeos, alguns autores têm se dedicado a usar bases de conhecimento para auxiliar na identificação de similaridade entre videoaulas e outros tipos de vídeos. Neste caso, é comum o uso de texto associado ao vídeo, como títulos, resumos e outros metadados, como também legendas ou tags criadas por usuários. Por exemplo, o trabalho de Chen, Cao, Song, Zhang, e Li (2010) que utiliza as categorias da Wikipedia para melhorar a categorização de vídeos identificando conceitos dos vídeos na Wikipedia através das tags e títulos criados por usuários. Dessa forma, os autores exploram as categorias associadas aos conceitos para fazer uma classificação desses vídeos. Contudo, os conceitos da 
Wikipedia são identificados manualmente a partir de títulos e tags de vídeos. Por outro lado, o trabalho de Raimond e Lowis (2012) utilizam transcrições automáticas do áudio de vídeos para identificar entidades na DBpedia e, com isso, associá-las aos vídeos. Os autores utilizam um modelo de espaço vetorial que possui a função de comparar a similaridade semântica entre expressões. Assim, as categorias da DBpedia são usadas para gerar interpretações para os termos presentes em um determinado transcrito a fim de identificar o contexto correto para as interpretações encontradas. Uma vez que as transcrições automáticas podem gerar muito ruído e as tags podem ser muito vagas, ambas as abordagens possuem problemas com acurácia.

Por fim, o cálculo de similaridade usando bases de conhecimento como apoio auxiliam em diversos tipos de sistemas. Se considerarmos especificamente a recuperação de vídeos educativos, o YoVisto (Sack \& Waitelonis, 2010) é o principal mecanismo de busca neste contexto. Este é um buscador que também utiliza ligações a dados externos para enriquecer as buscas. Sua especialidade está em vídeos acadêmicos e conferências. A principal contribuição que o YoVisto traz é o fato de usar a indexação do conteúdo com baixa granularidade, segmentando e estabelecendo tags por quadro ou trechos do vídeo. Para extrair informações esse buscador utiliza Folksonomia e técnicas de processamento de imagens, mais precisamente OCR (Optical Character Recognition) (Waitelonis, Sack, Hercher, \& Kramer, 2010). Estas informações são relacionadas com entidades da DBpedia para possibilitar ao motor de busca sugerir conceitos. Já o Iris AI (http://iris.ai), assim como o YoVisto, está voltado para o cenário acadêmico, onde sua função é a recomendação de artigos científicos a partir de uma indicação inicial do usuário. $\mathrm{O}$ Iris AI se aproxima da nossa proposta quando utiliza a similaridade entre documentos com uso de bases de conhecimento para relacioná-los. O sistema, por sua vez, usa um modelo de recomendação e processamento de linguagem natural a partir dos vídeos do repositório TED ${ }^{1}$ Talks para treinar esse modelo. Técnicas de inteligência artificial são parte fundamental do processo e necessita de bastante treinamento para conseguir alcançar boa acurácia.

\section{Anotação semântica}

Há tempos editores de conteúdo perceberam a necessidade de explicitar certos aspectos dos documentos a serem publicados. A essa tarefa foi atribuído o nome de anotação, ou seja, marcas colocadas nos documentos para instruir do que eram compostos com a finalidade de facilitar o entendimento e o assunto abordado nos mesmos (Ramalho, 2000).

As anotações podem ser feitas de forma manual ou de forma automática (Baeza-Yates \& Ribeiro-Neto, 2011). A anotação manual, mais custosa, normalmente é feita uma única vez, por ser exaustivo anotar muitas cenas (vídeos) ou parágrafos (texto). Uma alternativa menos custosa é a anotação automática. Algoritmos de anotação automática fazem uso de técnicas de processamento de linguagem natural para conseguir atribuir identificadores de conteúdo nos documentos analisados. Do ponto de vista da acurácia, anotações manuais tendem a ter melhor resultado, embora anotações automáticas tem alcançado também resultados significativos em diversos cenários.

Existem diversas técnicas para produzir anotação semântica (Oliveira \& Rocha, 2013; Slimani, 2013). Para anotação manual, é possível produzir anotações com a ajuda de editores, profissionais que são contratados para indexar informações aos conteúdos que possuem pouco ou nenhum texto prévio associado, como é o caso dos editores da BBC que são citados por Raimond e Lowis (2012). Ferramentas podem ajudar neste processo, como o VATIC ${ }^{2}$ para anotações manuais em vídeos.

\footnotetext{
http://www.ted.com

2 http://web.mit.edu/vondrick/vatic/
} 
Para anotações automáticas, algumas abordagens e ferramentas podem ser utilizadas. Para anotação de conteúdos textuais, como apostilas e textos didáticos, sistemas de extração de tópicos como o DBpedia Spotlight ${ }^{3}$ ou o Alchemy $^{4}$ podem ser utilizados com resultados próximos ao estado da arte. Como a anotação automática pode trazer muitos falsos positivos, as ferramentas de anotação geralmente permitem que o usuário determine a faixa de confiança dos resultados, fazendo com que a ferramenta tenda a atribuir poucos recursos por documento, mas com maior acurácia.

Para outras mídias como áudio, imagens e vídeos que possuem informação textual associada (descrição, título, legendas, entre outras), é comum realizar a anotação através apenas dessas informações textuais. O uso de tags livres associadas por usuários em aplicações web como blogs, Flickr e outros, pode ser utilizado como fonte para anotação, sendo este um exemplo clássico de folksonomia no processo de anotação (Aquino, 2007).

Quando informações não textuais não estão disponíveis em imagens, áudio ou vídeos, técnicas mais avançadas podem ser utilizadas, como o uso de OCR em imagens, PDFs ou vídeos (Vinciarelli, 2005), transcrição automática do áudio (Raimond \& Lowis, 2012), dentre outras.

O resultado do processo de anotação é um conjunto de identificadores de assuntos abordados no documento. As ferramentas mais atuais tem utilizado identificadores oriundos de alguma base de conhecimento na Web como anotadores. É o caso do DBpedia Spotlight e do Alchemy. $\mathrm{Na}$ nossa proposta, utilizaremos esses identificadores como forma de calcular similaridade entre os itens presentes em repositórios de recursos didáticos.

\section{Bases de conhecimento}

Utilizar fontes externas de conhecimento em sistemas de recuperação de informação já é uma realidade, pois a maior parte da informação disponível na web está no formato textual, o que não é o melhor formato para o processamento. Isso sugere a necessidade de obter essa informação em formato estruturado, a fim de chegar a uma interpretação objetiva e não ambígua. Uma maneira bem difundida para isso, é através dos dados ligados. Bizer, Heath, e Berners-Lee (2009) relatam que os dados ligados é um modo de organizar a web criando relações de acordo com o tipo de dado. Segundo Marx et al. (2014), dados ligados são fundamentais para agregar valor ao conjunto de dados original enriquecendo-o com associações a outros conjuntos de dados conexos. Neste sentido, a DBpedia ${ }^{5}$, projeto que extrai conhecimento estruturado, multilíngue da Wikipedia, destaca-se como uma das fontes de conhecimento mais populares e amplamente utilizada para consultas a dados ligados. A DBpedia define mais de 27 milhões de ligações RDF que apontam para mais de 30 fontes de dados externos. Isso faz com que cada vez mais a DBpedia se torne uma central de dados ligados, interligando outros centros de conexões em nuvem (Lehmann et al., 2014).

Algumas estruturas de conhecimento existentes, além de utilizarem fontes artesanais de alta qualidade, com conhecimento latente como a Wikipedia, utilizam também bases de conhecimento lexicográfico, como a WordNet. Exemplos dessas estruturas são o BabelNet (Navigli \& Ponzetto, 2010) e o LINDEN (Shen, Wang, Luo, \& Wang, 2012), que como resultado apresentam grandes volumes de entidades nomeadas em texto ligadas às bases de conhecimentos.

Analisando ainda projetos mais voltados para os dados estruturados, temos, como exemplo, o banco de dados FrameNet, o qual possui descrições de mais de 7.000 unidades lexicais com base em mais de 130.000 frases anotadas (Baker, Fillmore, \& Cronin, 2003), e o Freebase que

\footnotetext{
http://spotlight.dbpedia.org/

http://www.alchemyapi.com/

5 http://www.dbpedia.org/
} 
contém mais de 125.000.000 tuplas, mais de 4.000 tipos, e mais de 7.000 propriedades (Bollacker, Evans, Paritosh, Sturge, \& Taylor, 2008).

As grandes vantagens da utilização de bases de conhecimento está no fato dessas crescerem à medida que mais informações são inseridas, tornando assim o sistema que as utilizam mais específico e ao mesmo tempo mais abrangente. A possibilidade de realizar inferências através desse tipo de base também se torna um grande atrativo. Um exemplo disso seria: uma consulta de todos os seriados em que dois atores aparecem, se essa consulta retorna 3 seriados, podemos inferir que esses seriados são relacionados ao menos pelo fato de possuírem o mesmo elenco (Mirizzi, Di Noia, Ragone, Ostuni, \& Di Sciascio, 2012).

Quando essas possibilidades são inseridas na recomendação é factível obter resultados relevantes como o aumento de relações entre vídeos à medida que as bases crescem; a descoberta de relações que não estão explícitas. Fatores que viabilizam o dinamismo de qualquer sistema que as utilizem.

Por esses e outros motivos essas bases de conhecimentos aqui apresentadas, estão presentes na literatura como soluções efetivas. Em relação a essa proposta, quaisquer dessas bases poderiam ser utilizadas. Como será visto na seção seguinte, nossa restrição é que as bases contenham um grafo de categorias. Utilizamos a DBpedia neste trabalho por ser uma das bases mais importantes atualmente. Além disso, o conteúdo enciclopédico da Wikipedia/DBpedia se mostra adequado para calcular similaridade em repositórios de conteúdos educacionais por possuir boa cobertura nas principais áreas de conhecimento e ter profundidade nos principais tópicos dentro dessas áreas (Halavais \& Lackaff, 2008). Para repositórios com conteúdos mais específicos e sem tanta cobertura da DBpedia, deve-se considerar o uso de outras bases ou ontologias mais adequadas aos tópicos abordados no repositório.

\section{Abordagem para predição de relacionamentos entre vídeos}

A fim de encontrar relação entre os vídeos dentro de um repositório de videoaulas, vamos construir um procedimento que enriquece sistemas de classificação existentes, por meio da predição de videoaulas, baseado no contexto semântico de cada vídeo. Para isso, utilizaremos o conteúdo estruturado das informações da DBpedia.

Considerando apenas as propriedades que serão usadas na solução, pode-se definir um vídeo $v_{i}$ como uma tupla $v_{i}=<r_{1}, r_{2}, r_{3}, \ldots, r_{n}>$ onde $r_{j}$ são recursos da DBpedia, ou seja, URIs que identificam pessoas, lugares, e organizações dentro da DBpedia. Os recursos $r_{j}$ podem ser considerados metadados do vídeo que foram associados manualmente ou através de um processo de anotação semântico, como em (Habibian, Mensink, \& Snoek, 2015; Ramalho, 2000; Sack \& Waitelonis, 2010).

No nosso trabalho, um recurso é um identificador da DBpedia, a qual corresponde a uma página da Wikipedia que descreve um assunto qualquer como, por exemplo, "calor" ou "algoritmos". Esse recurso da DBpedia pode ser formalizado como uma tupla $r_{j}=<u r i_{j}, L\left(r_{j}\right), C\left(r_{j}\right), P\left(r_{j}\right)>$, onde $u r i_{j}$ representa o identificador do recurso, $L\left(r_{j}\right)$ o conjunto dos nomes (label) que esse recurso possui, $C\left(r_{j}\right)$ o conjunto de categorias desse recurso e $P\left(r_{j}\right)$ o conjunto de propriedades do recurso.

Em repositórios de vídeos educacionais em língua portuguesa, é comum encontrar metadados direcionando para recursos em português. Assim, é esperado que se faça uso da versão em português da DBpedia, denominada DBpediaPT ${ }^{6}$. Neste cenário, é importante ressaltar que o

${ }^{6} \mathrm{http}: / /$ www.dbpedia.pt/ 
volume de informações no repositório em português é muito menor que no repositório em inglês da DBpedia. Assim, utilizamos as duas bases em conjunto e navegamos entre as bases para garantir que seja recuperado o maior número de recursos possíveis. Para tal, considere a função $f\left(r_{j}\right.$,@en) sendo a função que retorna o recurso $r_{j}^{\prime}$ na DBpedia EN correspondente ao recurso $r_{j}$ da DBpedia PT. Para chegar a esse resultado nessa função, usamos a propriedade owl:sameAs.

A partir de então, consideramos as seguintes definições para este processo, $\mathrm{R}$ é um conjunto de todos os recursos associados a um vídeo onde $r_{j}$ é um recurso específico, ex.: $<$ http://pt.dbpedia.org/resource/Luz>. Quando usamos a função $f\left(r_{j}\right.$, @en), obtemos o recurso correspondente na DBpedia inglês, ex.: <http://dbpedia.org/resource/Light $>$. Como o conjunto de todas as categorias associadas a um desses recursos específico temos $C\left(r_{j}^{\prime}\right)$. Por exemplo, para o recurso Light, temos: $\mathrm{C}(\mathrm{http} / / \mathrm{dbpedia}$. org/resource/Light $)=$ \{http://dbpedia.org/resource/Category:Light\}.

Expandimos então nosso conjunto de categorias, pegando as categorias $C^{+}\left(r_{j}^{\prime}\right)$, que é o conjunto de todas as categorias associadas como mais abrangentes que alguma categoria $c \in C\left(r_{j}^{\prime}\right)$. Assim, temos que $C^{+}\left(r^{\prime}{ }_{j}\right)=\left\{x \mid x \in u p(c, \alpha) \wedge c \in C\left(r_{j}^{\prime}\right)\right\}$, onde up(c, i) representa as categorias mais abrangentes que c até uma distância $\alpha$ de c no grafo. Para isso consultamos a propriedade skos:broader. Por exemplo, para o recurso Light, temos: $C^{+}$(http://dbpedia.org/resource/Light) $=\{$Electrodynamics, Electromagnetic_radiation, Electromagnetic_spectrum, Optics, Wave\}.

De forma semelhante, expandimos ainda mais nosso conjunto de categorias, pegando também as categorias $C^{-}\left(r_{j}^{\prime}\right)$, que é o conjunto de todas as categorias associadas como uma categoria menos abrangente que uma categoria $c \in C\left(r_{j}^{\prime}\right)$. Formalmente, $C^{-}\left(r^{\prime}{ }_{j}\right)=\left\{x \mid x \in \operatorname{down}(c, \beta) \wedge c \in C\left({r^{\prime}}_{j}\right)\right\}$, onde down(c, i) representa todas as categorias menos abrangentes que $\mathrm{c}$ até uma distância $\beta$ de $\mathrm{c}$ no grafo. Então consultamos a propriedade skos:broader_of, como, por exemplo, $C^{-}(\mathrm{http}: / \mathrm{dbpedia.org} /$ resource/Light $)=\{$ Darkness, Light_sources, Lighting, Photons, Vision, Photochemistry, Light_therapy, Fictional_characters_who_can_manipulate_light\}

Portanto, obtemos o conjunto de todas as categorias encontradas para $r_{j}^{\prime}$, como sendo $\varphi\left(r_{j}^{\prime}\right)=C^{+}\left(r_{j}^{\prime}\right) \cup C\left(r_{j}^{\prime}\right) \cup C^{-}\left(r_{j}^{\prime}\right)$. Assim, a lista de categorias relacionadas a um vídeo é dado por $\Upsilon(v)=\bigcup_{r \in v} \varphi(r)$. Então, concluímos que os vídeos $v_{i}$ e $v_{j}$ serão relacionados se $\operatorname{sim}\left(v_{i}, v_{j}\right)>\alpha$, sendo $\alpha \in[0,1]$ um valor pré determinado. A similaridade entre dois vídeos pode ser calculada como (Equação 1):

$$
\operatorname{sim}\left(v_{i}, v_{j}\right)=\frac{\left|\Upsilon\left(v_{i}\right) \cap \Upsilon\left(v_{j}\right)\right|}{\left|\Upsilon\left(v_{i}\right) \cup \Upsilon\left(v_{j}\right)\right|}
$$

A predição de relacionamentos se dá através do cômputo da similaridade de tal forma que os vídeos com maior valor de similaridade serão acrescidos deste relacionamento.

A Figura 1 apresenta, de forma mais simplificada, as relações entre vídeos, recursos e categorias. Neste exemplo, temos que $v_{1}=<r_{1}, r_{4}>, v_{2}=<r_{2}, r_{3}>$ e $v_{3}=<r_{1}, r_{3}, r_{5}>$. Verificando em relação a $v_{3}$, este compartilha exatamente um recurso com $v_{1}$ e um recurso com $v_{2}$. Contudo, ao analisar as categorias associadas aos recursos, verifica-se que o vídeo $v_{3}$ possui mais categorias em comum a $v_{1}$ que a $v_{2}$. 


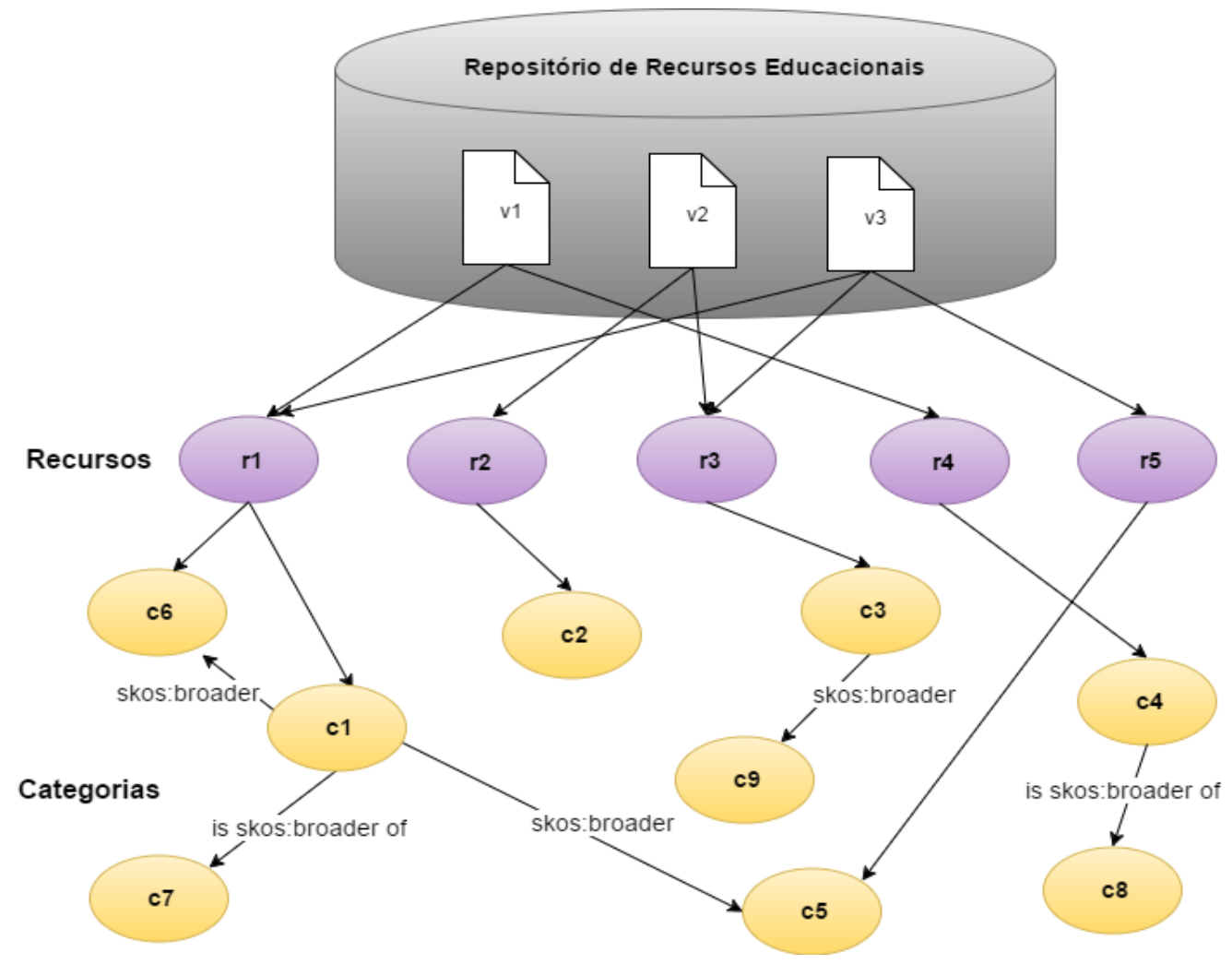

Figura 1: Ligações entre conceitos e categorias na DBpedia.

Para melhor compreensão da proposta, o algoritmo abaixo representa todo o processo:

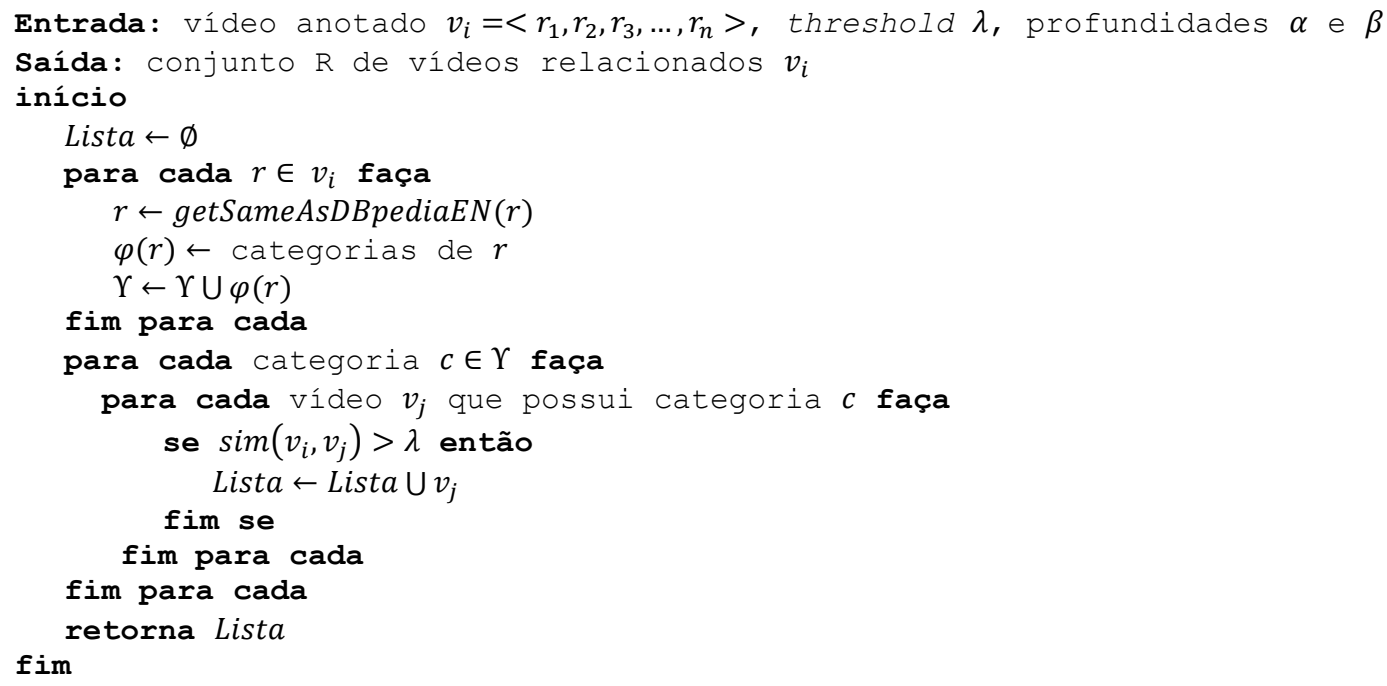

Código 1: Algoritmo do processo realizado. 


\section{Avaliação da abordagem}

Para avaliação da abordagem foi criada uma base de vídeos com relacionamentos manuais definidos por especialistas da área de Ciências Exatas. Nesta base, para cada vídeo foi informado o relacionamento deste com os demais vídeos existentes no repositório. Esperávamos que a abordagem encontrasse novos relacionamentos além dos existentes.

A base de avaliação possui 41 vídeos, sendo esses das áreas de Ciência da Computação, Estatística, Química e Física. A base foi populada com todas as videoaulas do Instituto de Ciências

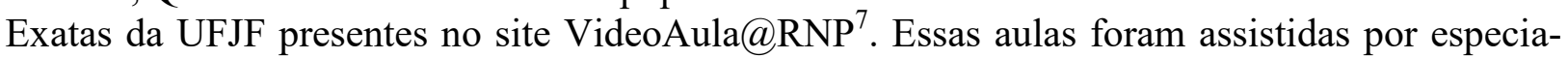
listas convidados a realizar duas tarefas. Primeiro, cada especialista atribuiu um recurso da DBpedia para cada assunto explicitamente falado durante o vídeo. Após assistir um conjunto de vídeos, cada especialista informou quais os vídeos mais relacionados a cada vídeo assistido. Não houve restrição do número de recursos e da quantidade de relações para cada vídeo que o especialista poderia atribuir. Ainda, a seleção dos vídeos relacionados seguiu critérios pessoais de cada especialista. Como a definição dos critérios seguiram critérios pessoais, os relacionamentos finais são a união dos relacionamentos atribuídos por cada especialista, não tendo sido utilizada nenhuma filtragem posterior. Assim, um especialista pode considerar que um vídeo é relacionado por abordar exatamente um mesmo tópico de aula e outro especialista pode relacionar outro vídeo por considera-lo complementar a um pequeno assunto abordado durante a aula. No total foram definidos manualmente 211 relacionamentos, com uma média de 5 relacionamentos por vídeo. Nenhum dos vídeos da base ficou sem relacionamento, apesar de existirem 10 vídeos com apenas 1 relacionamento, 17 vídeos tiveram 5 relacionamentos ou mais.

Depois de caracterizado nosso repositório, definimos como métricas de avaliação a cobertura (recall) e o TopN.

A cobertura foi calculada através da seguinte fórmula (Equação 2):

cobertura $=\frac{\sum(\text { itens_relevantes } \cap \text { itens_recuperados })}{\sum(\text { itens_relevantes })}$

A cobertura é máxima, ou seja, igual a 1, quando todos os itens relevantes são retornados, cobrindo todos os resultados esperados, e mínima, igual a 0, quando nenhum item relevante é retornado. Como nosso objetivo era relacionar o maior número possível de vídeos com assuntos em comum, esperávamos maximizar a cobertura.

A métrica TopN fornece o quão bem posicionados estão os itens relevantes. Ele será máximo, ou seja, igual a 1, se todos os itens relevantes são retornados e estão exatamente nas primeiras posições. Por outro lado, um TopN igual a 0 significa que nenhum item relevante foi retornado. Admitindo-se que a base manual está correta, a melhor situação é aquela em que a busca obtém cobertura e TopN máximos.

Para calcular o TopN os itens retornados foram ranqueados considerando o número de categorias em comum que possuem com o vídeo inicial. Quanto maior for esse número, mais correlatos são os assuntos tratados nos vídeos, justificando uma melhor colocação no ranking. $\mathrm{O}$ TopN foi calculado através da seguinte fórmula (Equação 3), adaptada de (Berenzweig, Logan, Ellis, \& Whitman, 2004):

$$
\operatorname{TopN}=\frac{\sum_{\mathrm{j}=1}^{\mathrm{N}} \omega^{\mathrm{k}_{\mathrm{j}}}}{\sum_{\mathrm{i}=1}^{\mathrm{N}} \omega^{\mathrm{i}}}
$$

\footnotetext{
${ }^{7}$ http://www.videoaula.rnp.br
} 
$\mathrm{Na}$ fórmula acima, $\mathrm{N}$ é o número de vídeos manualmente relacionados pelos especialistas, kj é a posição do vídeo j no resultado dos vídeos automaticamente relacionados e $0<\omega<1$ é uma constante de decaimento, a qual expressa o quanto se quer penalizar um vídeo por aparecer em posições inferiores no resultado. Para o estudo foi escolhido $\omega=0.8$, o que significa que um vídeo irá contribuir com aproximadamente 0.1 no resultado final antes da normalização se ele ocorrer na décima colocação do resultado ranqueado.

\subsection{Experimentos e resultados}

Nos experimentos realizados, foram alterados os parâmetros alfa e beta do algoritmo de forma a recuperar diferentes níveis de informações de categorias da DBpedia em cada um dos experimentos. Assim, pode-se analisar como a quantidade de informação processada influencia na acurácia do processo e no tempo de processamento. Os resultados alcançados nas métricas de cobertura e TopN em cada experimento estão descritos nas Tabelas 1 e 2, respectivamente.

Tabela 1: Média da cobertura em cada experimento.

\begin{tabular}{|c|c|c|c|}
\hline $\boldsymbol{\alpha} \backslash \boldsymbol{\beta}$ & $\mathbf{0}$ & $\mathbf{1}$ & $\mathbf{2}$ \\
\hline $\mathbf{0}$ & 0,67946 & 0,91412 & 0,93120 \\
\hline $\mathbf{1}$ & 0,91800 & 0,93120 & 0,93120 \\
\hline $\mathbf{2}$ & 0,93120 & 0,93120 & 0,93120 \\
\hline
\end{tabular}

Tabela 2: Média do TopN em cada experimento.

\begin{tabular}{|c|c|c|c|}
\hline $\boldsymbol{\alpha} \backslash \boldsymbol{\beta}$ & $\mathbf{0}$ & $\mathbf{1}$ & $\mathbf{2}$ \\
\hline $\mathbf{0}$ & 0,55103 & 0,66778 & 0,66776 \\
\hline $\mathbf{1}$ & 0,66761 & 0,67732 & 0,63420 \\
\hline $\mathbf{2}$ & 0,61300 & 0,62910 & 0,60832 \\
\hline
\end{tabular}

Nos resultados alcançados, verifica-se que caminhar em categorias mais abrangentes (alfa) e mais específicas (beta) produzem maior cobertura. Porém, a cobertura não muda quanto maior for a profundidade. Por outro lado, embora o uso das categorias possa ajudar em um melhor TopN, aumentar a profundidade não implica em aumento do TopN. Ao aumentar a profundidade, mais categorias em comum dos vídeos terão e, com isso, mais difícil será ranquear corretamente o resultado através do número de categorias em comum. Este efeito é mais evidente ao caminhar com maior profundidade nas categorias mais abrangentes (alfa $=2)$. A melhor configuração encontrada para o algoritmo foi alfa $=1$ e beta $=1$.

Para uma visão mais detalhada dos resultados, o gráfico 1 apresenta os valores de cobertura e TopN (eixo Y) para cada vídeo da base de avaliação (eixo X). Neste gráfico, os vídeos foram ordenados de forma decrescente por TopN para permitir uma visão individual mais acurada dos testes em cada vídeo. Assim o leitor pode identificar com maior precisão a proporção de vídeos com resultados bons e ruins em cada experimento. Verifica-se nos gráficos 1A, 1B e 1C que a cobertura aumenta conforme mais informações são processadas pelo algoritmo. Por exemplo, no gráfico $1 \mathrm{~A}$, temos 9 vídeos que resultaram em uma cobertura máxima (vídeos-testes numerados de 1 a 9) enquanto no gráfico $1 \mathrm{~B}$, houveram 20 vídeos com cobertura máxima. Ao utilizar categorias amplas e específicas com alfa $=1$ e beta $=1$ (Gráfico 1C), houve uma cobertura próxima de 0,9 em 32 vídeos. Em relação ao TopN, verifica-se que o algoritmo conseguiu retornar os vídeos corretos como primeiros resultados, ou seja, próximo ao topo do ranqueamento (TopN alto), em mais da metade da base de avaliação. É possível verificar também que os valores de TopN seguem a tendência de melhoria quanto mais informações são processadas pelo algoritmo. Verifica-se também que o processamento de categorias específicas (Gráfico 1B) é capaz de apresentar um resultado melhor em relação à abordagem sem expansão (Gráfico 1A). Isto se dá por conta da rede de categorias da DBpedia, a qual permite que um recurso esteja ligado a mais de uma categoria e que uma dada categoria possa estar ligada (por abrangência ou especificidade) a várias outras categorias. Assim, dois vídeos, cada um ligado a uma categoria distinta, podem ser considerados com algum grau de similaridade se estas categorias são, por exemplo, subcategorias umas das outras ou se possuem outras subcategorias (ou supercategorias) em comum. 
Como os resultados são ranqueados para cada vídeo, é possível utilizar um método de poda (threshold) no resultado para retornar apenas um conjunto limitado de vídeos no conjunto resultante, mas de alta precisão. O Gráfico 1D apresenta os resultados do terceiro experimento retirando do resultado todos os vídeos que não tivessem ao menos 10 categorias em comum. Verifica-se que o método de poda não influenciou consideravelmente o resultado do algoritmo, visto que os valores de TopN já indicavam um bom ranqueamento do resultado. Por outro lado, como é de se esperar, a cobertura tende a cair quando utilizamos um método de poda no conjunto resultante. A cobertura, contudo, cai muito pouco quando realizamos o experimento com o método de poda. Em relação à média, o método de poda neste experimento fez o TopN subir de 0,67732 para 0,68215 e a cobertura cair de 0,93120 para 0,87715 .

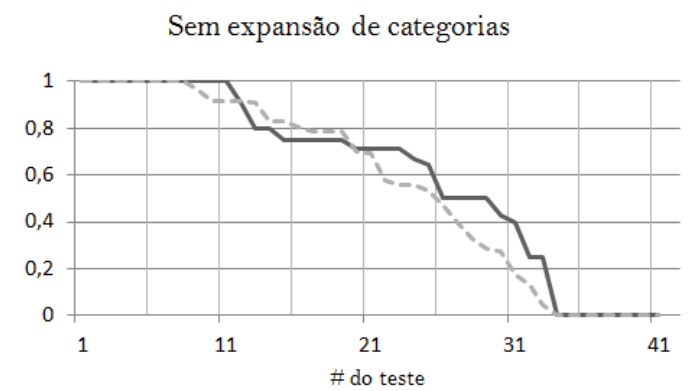

(A)

Expandindo por categorias amplas e específicas (sem poda)

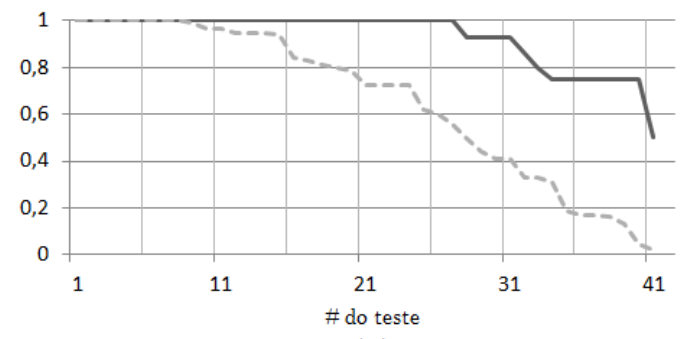

$(\mathrm{C})$
Expandindo por categorias mais específicas

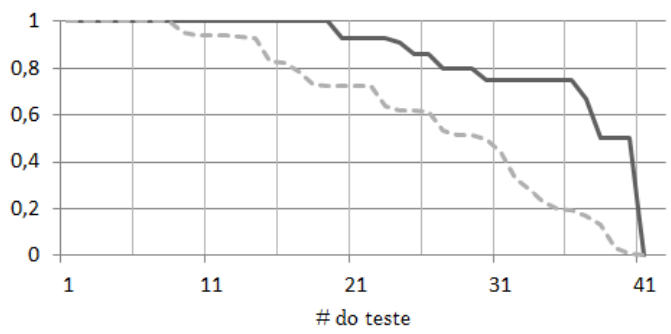

(B)

Expandindo por categorias amplas e específicas (com poda)

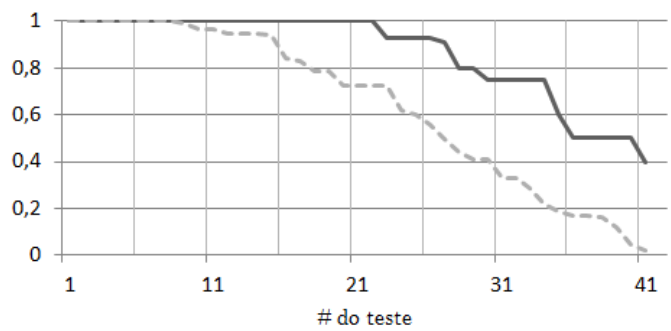

(D)

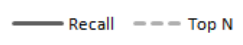

Gráfico 1 : Valores de cobertura (linha contínua) e TopN (linha tracejada) para cada experimento realizado.

Em relação ao experimento com alfa e beta iguais a 1, o Gráfico 2 apresenta a dispersão da cobertura em relação ao TopN em relação a base de teste. Verifica-se que, numa faixa de cobertura, os valores de TopN podem variar em até 20 pontos percentuais. Assim, pode-se verificar uma tendência no algoritmo, onde quanto maior a cobertura do resultado, melhor tende a ser o ranqueamento dos vídeos.

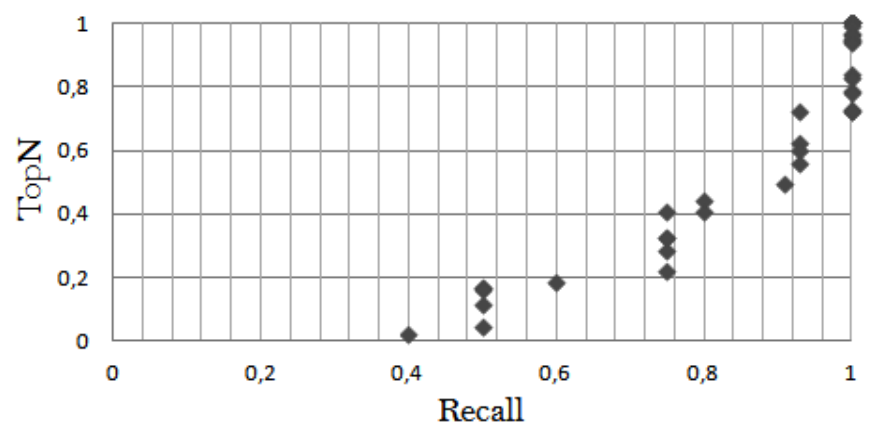

Gráfico 2: Dispersão da cobertura e TopN no experimento com poda. 
Por fim, o custo de processamento do algoritmo foi verificado para cada experimento e os resultados estão apresentados no Gráfico 3. Para avaliação, foram registrados os tempos de processamento utilizando o endpoint SPARQL remoto da DBpedia, de forma a verificar que a abordagem é viável de ser utilizada diretamente no dado mais atual da base de conhecimento e sem necessidade de pré-processamento local. O gráfico registra a duração de processamento de todo o benchmark. O maior tempo de processamento se dá quando são analisadas as categorias mais abrangentes (alfa), uma vez que um recurso pode estar associado a um número grande de categorias e essas categorias, por sua vez, podem estar ligadas a diversas outras no grafo da DBpedia. Por outro lado, o número de nós alcançados ao caminhar pelas categorias mais específicas (beta) tenda a ser menor porque as anotações tendem a apontar para recursos que representam conceitos mais específicos, os quais geralmente não possuem tantas ramificações quanto seus ancestrais. Por exemplo, é mais provável encontrar um vídeo anotado com um conceito Juiz_de_Fora do que Distrito_administrativo.

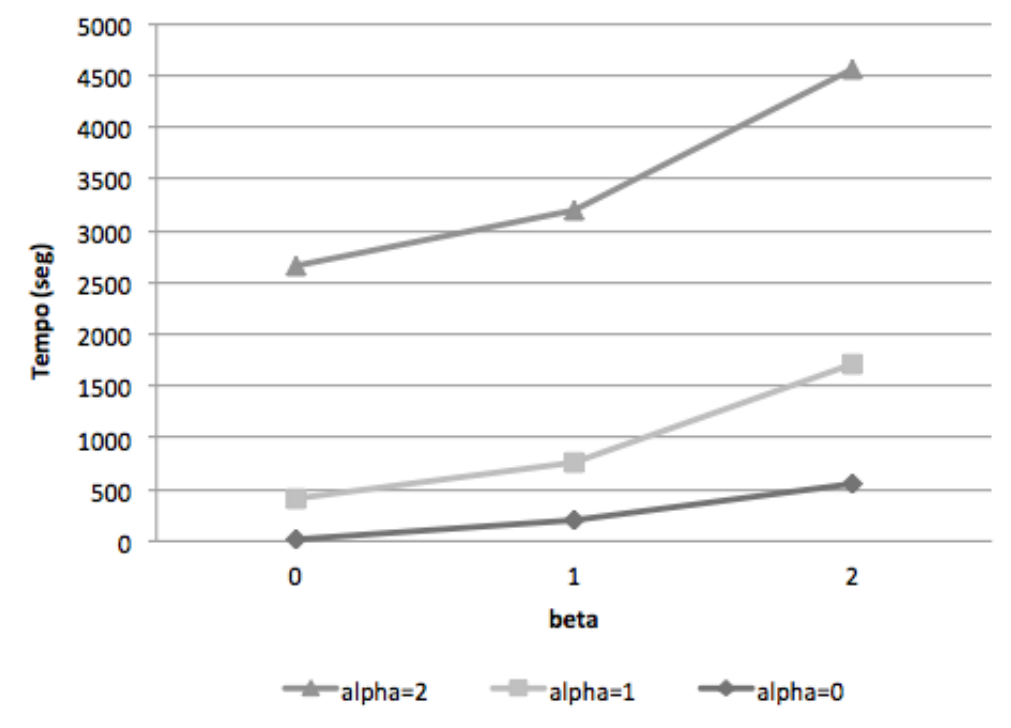

Gráfico 3: Tempo de processamento do algoritmo para cada valor de alfa e beta.

\subsection{Análise dos falsos positivos}

Embora os resultados dos experimentos tenham sido satisfatórios, é importante analisar os falsos positivos gerados pelo algoritmo para a base de avaliação. A base de avaliação foi criada manualmente por especialistas seguindo critérios pessoais para determinar quais vídeos deveriam ser relacionados. Como resultado, é possível identificar vídeos na base com poucos relacionamentos ou vídeos que foram identificados pela maioria dos especialistas como relacionados mas que não possuem nenhum recurso em comum. Dessa forma, a base de avaliação apresenta relações e atribuição de recursos que não estão enviesadas por algum conjunto de informações ou métodos de seleção.

Levando em consideração estas particularidades, os experimentos realizados mostraram que os relacionamentos falsos positivos que possuem um grande número de categorias em comum com o vídeo analisado são, na sua grande maioria, relações que estão ausentes na base de avaliação. Ou seja, o algoritmo é capaz de identificar relacionamentos que nem sempre são facilmente identificáveis por uma pessoa. Tome, por exemplo, o vídeo identificado como "fis2tempcalor", o qual aborda conceitos de temperatura e calor e foi relacionado manualmente apenas com o vídeo identificado como "fis2cap18-parte2". O algoritmo, por sua vez, relacionou 15 vídeos com "fis2tempcalor", entre elas aulas de conteúdos de física e química que abordam de conceitos indiretamente relacionados com temperatura e calor. 
Outros exemplos como o acima foram encontrados no experimento, o que não invalida a base de avaliação, mas apenas deixa claro que alguns resultados podem ser classificados como falsos positivos devido à subjetividade da construção manual da base de avaliação, o que, de certa forma, a aproxima de um cenário real.

\section{Conclusões e Trabalhos Futuros}

O aumento de informação disponível em repositórios de conteúdos educacionais é visível e a necessidade de encontrar conteúdo relevante nas buscas, principalmente em vídeo, onde as informações não estão tão explícitas como nos textos, justifica esta pesquisa.

Neste trabalho utilizamos uma abordagem que utiliza dados ligados para estabelecer relações que não estão explícitas em recursos anotados. Nossa principal fonte de informação foi a DBpedia. Utilizamos os relacionamentos descritos em sua ontologia para expandirmos os recursos anotados nos documentos de um repositório e, assim, verificar a similaridade entre diferentes documentos. Desta forma, documentos distintos podem ser identificados como similares mesmo se não possuem as mesmas anotações. Relacionamentos entre estes documentos podem ser feitos automaticamente, usando conceitos que nem sempre um usuário relacionaria.

De acordo com os resultados apresentados neste artigo, concluímos que nossa abordagem é satisfatória, uma vez que os resultados apresentam aproximadamente $90 \%$ de cobertura dos itens relevantes; os vídeos retornados estão bem colocados no ranking conforme indica os valores de TopN; mesmo para os vídeos com pouca informação inicial foi possível estabelecer relações; vídeos totalmente diferentes dificilmente serão relacionados; e por fim, não é necessário o uso de esforço manual para a classificação dos vídeos, já que os mesmos podem passar por abordagens que permitem a categorização automática, como em (Coelho \& De Souza, 2014). A base de avaliação utilizada nos experimentos está disponível no github ${ }^{8}$ e pode ser útil para outros pesquisadores.

Como contribuição para repositórios de objetos de aprendizagem e portais que disponibilizam grandes volumes de conteúdos educacionais com forte presença da mídia vídeo (videoaulas), o trabalho permite, ao identificar objetos similares através das anotações semânticas presentes nestes objetos, que mecanismos de busca possam expandir uma consulta através da inclusão de recursos relacionados com os documentos retornados ou que documentos relacionados possam ser recomendados de acordo com o documento visualizado por um usuário ou com o histórico de visualizações do usuário. Ainda, ao utilizar bases externas para identificar documentos similares, é possível que os resultados tendam a melhorar conforme mais informações sejam acrescentadas nestas bases.

Para trabalhos futuros pretende-se analisar a abrangência de outras bases de conhecimento para o problema de recomendação, estudar uma forma de tratar o uso de diferentes bases de conhecimento simultaneamente e incluir essa abordagem de classificação em um sistema de recomendação de vídeo levando em consideração o perfil do usuário. Ainda, o uso simultâneo de diversas bases de conhecimento gera o problema técnico de processamento desse volume de dados, sendo necessário melhorar a abordagem para realizar filtros durante o processo de busca das categorias. Por fim, um experimento com participação de especialistas poderia avaliar o grau de novidade (Vargas \& Castells, 2011) que o algoritmo possui na base de avaliação. Dessa forma, é possível avaliar a quantidade de resultados atualmente classificados como falsos positivos que são, na verdade, relacionamentos corretos não atribuídos anteriormente na base de avaliação.

\footnotetext{
${ }^{8}$ https://github.com/ufjf-dcc/LAPIC1-benchmark
} 


\section{Agradecimentos}

Agradecemos a RNP (Rede Nacional de Ensino e Pesquisa) e a CAPES (Coordenação de Aperfeiçoamento de Pessoal de Nível Superior) pelo financiamento desse trabalho.

\section{Referências}

Aquino, M. C. (2007). Hipertexto 2.0, folksonomia e memória coletiva: um estudo das tags na organização da web. E-Compós, Brasília, 9. [GS search]

Araújo, L. D. R., \& de Souza, J. F. (2011). Aumentando a transparência do governo por meio da transformação de dados governamentais abertos em dados ligados. Revista Eletrônica de Sistemas de Informação, 10(1). doi: 10.5329/RESI.2011.1001007 [GS search]

Baeza-Yates, R., \& Ribeiro-Neto, B. (2011). Modern Information Retrieval - the concepts and technology behind search, (2a ed.). Pearson Education Ltd., Harlow, England. [GS search]

Baker, C. F., Fillmore, C. J., \& Cronin, B. (2003). The structure of the FrameNet database. International Journal of Lexicography, 16(3), 281-296. doi: 10.1093/ij1/16.3.281. [GS search]

Barrére, E. (2014). Videoaulas: aspectos técnicos, pedagógicos, aplicações e bricolagem. Anais da $3^{a}$ Jornada de Atualização em Informática na Educação, 3(1). [GS search]

Berenzweig, A., Logan, B., Ellis, D. P., \& Whitman, B. (2004). A large-scale evaluation of acoustic and subjective music-similarity measures. Computer Music Journal, 28(2), 63-76. doi: $\underline{10.1162 / 014892604323112257}$. [GS search]

Bizer, C., Heath, T., \& Berners-Lee, T. (2009). Linked data-the story so far. Semantic Services, Interoperability and Web Applications: Emerging Concepts, (pp. 205-227). doi: 10.4018/jswis.2009081901. [GS search]

Bollacker, K., Evans, C., Paritosh, P., Sturge, T., \& Taylor, J. (2008, June). Freebase: a collaboratively created graph database for structuring human knowledge. In Proceedings of the 2008 ACM SIGMOD international conference on Management of data (pp. 1247-1250). doi: $\underline{10.1145 / 1376616.1376746}$.[GS search]

Casagrande, M. F. R., Kozima, G., \& Willrich, R. (2015). A Recommendation Technique Based on Metadata for Digital Repositories Oriented to Learning. Brazilian Journal of Computers in Education, 23(02), 70. doi: 10.5753/rbie.2015.23.02.70. [GS search]

Chen, Z., Cao, J., Song, Y., Zhang, Y., \& Li, J. (2010, October). Web video categorization based on Wikipedia categories and content-duplicated open resources. In Proceedings of the 18th ACM international conference on Multimedia (pp. 1107-1110). doi: 10.1145/1873951.1874162. [GS search]

Cheniki, N., Belkhir, A., Sam, Y., \& Messai, N. (2016, June). LODS: A Linked Open Data Based Similarity Measure. In Enabling Technologies: Infrastructure for Collaborative Enterprises (WETICE), 2016 IEEE 25th International Conference on (pp. 229-234). doi: 10.1109/WETICE.2016.58. [GS search]

Coelho, S. A., \& de Souza, J. F. (2014). Anotação semântica de transcritos para indexação e busca de vídeos. In Conferência Ibero Americana (WWW/INTERNET). [GS search]

Cunha, M., \& Faustino, J. C. (2014). Vídeo@ RNP: integrando serviços de vídeo digital para a comunidade acadêmica. Cuarta Conferencia de Directores de Tecnología de Información, TICAL2014 Gestión de las TICs para la Investigación y la Colaboración, Cancún. [GS search] 
Da Motta, C. L. R., \& Lopes, L. M. C. (2002). Sistema de Recomendação apoiando a TVEscola. In Anais do Simpósio Brasileiro de Informática na Educação, 1(1), 377-384. doi: 10.5753/cbie.sbie.2002.377-384. [GS search]

Dasiopoulou, S., Giannakidou, E., Litos, G., Malasioti, P., \& Kompatsiaris, Y. (2011). A survey of semantic image and video annotation tools. In Knowledge-driven multimedia information extraction and ontology evolution (pp. 196-239). doi: 10.1007/978-3-642-20795-2_8 [GS $\underline{\text { search] }}$

Davidson, J., Liebald, B., Liu, J., Nandy, P., Van Vleet, T., Gargi, U., ... \& Sampath, D. (2010, September). The YouTube video recommendation system. In Proceedings of the fourth ACM conference on Recommender systems (pp. 293-296). doi: 10.1145/1864708.1864770. [GS search]

De Oliveira, F. K., Santana, J. R., \& de Oliveira Pontes, M. G. (2010). O vídeo como ferramenta educacional a partir de múltiplas plataformas. In Brazilian Symposium on Computers in Education (Simpósio Brasileiro de Informática na Educação-SBIE), 1(1). doi: 10.5753/cbie.sbie.2010.\%25p. [GS search]

Diana, J. B., Giuffra, C. E., Nakayama, M. K., Spanhol, F. J., \& Silveira, R. A. (2015). O uso dos repositórios na visão do Designer Instrucional. Revista Brasileira de Informática na Educação, 23(01), 173. doi: 10.5753/rbie.2015.23.01.173. [GS search]

Figueiredo, A. P. S., Assireu, A. T., \& Souza, V. C. O. (2015). Material didático multimídia aplicado a educação semi-presencial: um relato de experiência na graduação. In: Revista Brasileira de Informática na Educação, 23 (2), 128-136. [GS search]

Gupta, Y., Saini, A., \& Saxena, A. K. (2015). A new fuzzy logic based ranking function for efficient information retrieval system. Expert Systems with Applications, 42(3), 1223-1234. doi: 10.1016/j.eswa.2014.09.009. [GS search]

Habibian, A., Mensink, T., \& Snoek, C. G. (2015, June). Discovering Semantic Vocabularies for Cross-Media Retrieval. In Proceedings of the 5th ACM on International Conference on Multimedia Retrieval (pp. 131-138). doi: 10.1145/2671188.2749403. [GS search]

Halavais, A., \& Lackaff, D. (2008). An analysis of topical coverage of Wikipedia. Journal of Computer -Mediated Communication, 13(2), 429-440. doi: 10.1111/j.10836101.2008.00403.x. [GS search]

Herrera, J. E. T., Casanova, M. A., Nunes, B. P., Lopes, G. R., \& Leme, L. A. P. P. (2016). DBpedia Profiler Tool: Profiling the Connectivity of Entity Pairs in DBpedia. In Proceedings of the 5th International Workshop on Intelligent Exploration of Semantic Data (IESD 2016). [GS search]

Hulpus, I., Hayes, C., Karnstedt, M., \& Greene, D. (2013, February). Unsupervised graph-based topic labelling using dbpedia. In Proceedings of the sixth ACM international conference on Web search and data mining (pp. 465-474). doi: 10.1145/2433396.2433454. [GS search]

IRIS AI. Iris AI Science Assistant. Recuperado em 11 outubro, 2016, de http://iris.ai

Jain, P., Hitzler, P., Sheth, A. P., Verma, K., \& Yeh, P. Z. (2010, November). Ontology alignment for linked open data. In International Semantic Web Conference (pp. 402-417). doi: 10.1007/978-3-642-17746-0_26. [GS search]

Jiang, Y. G., Bhattacharya, S., Chang, S. F., \& Shah, M. (2013). High-level event recognition in unconstrained videos. International journal of multimedia information retrieval, 2(2), 73101. doi: 10.1007/s13735-012-0024-2. [GS search] 
Kawase, R., Siehndel, P., Pereira Nunes, B., Herder, E., \& Nejdl, W. (2014, September). Exploiting the wisdom of the crowds for characterizing and connecting heterogeneous resources. In Proceedings of the 25th ACM conference on Hypertext and social media (pp. 56-65). doi: 10.1145/2631775.2631797. [GS search]

Köhncke, B., \& Balke, W. T. (2010, October). Using wikipedia categories for compact representations of chemical documents. In Proceedings of the 19th ACM international conference on Information and knowledge management, (pp. 1809-1812). doi: 10.1145/1871437.1871735. [GS search]

Lehmann, J., Isele, R., Jakob, M., Jentzsch, A., Kontokostas, D., Mendes, P. N., ... \& Bizer, C. (2014). DBpedia-a large-scale, multilingual knowledge base extracted from Wikipedia. Semantic Web Journal. doi: DOI: 10.3233/SW-140134. [GS search]

Marx, E., Usbeck, R., Ngomo, A. C. N., Höffner, K., Lehmann, J., \& Auer, S. (2014, September). Towards an open question answering architecture. Proceedings of the 10th International Conference on Semantic Systems, (pp. 57-60). doi: 10.1145/2660517.2660519. [GS search]

McGreal, R. (2004). Learning objects: A practical definition. International Journal of Instructional Technology and Distance Learning (IJITDL), 9(1). [GS search]

Medeiros, S. F. L., \& Pansanato, L. T. E. (2015, October). Estudo das Preferências de Alunos e Professores sobre Videoaula para Identificar Requisitos de Software para Ferramentas de Produção. In Brazilian Symposium on Computers in Education (Simpósio Brasileiro de Informática na Educação-SBIE) (Vol. 26, No. 1, p. 219). doi: 10.5753/cbie.sbie.2015.219. [GS search]

Mendes, P. N., Jakob, M., García-Silva, A., \& Bizer, C. (2011, September). DBpedia spotlight: shedding light on the web of documents. In Proceedings of the 7th international conference on semantic systems (pp. 1-8). doi: 10.1145/2063518.2063519. [GS search]

Mirizzi, R., Di Noia, T., Ragone, A., Ostuni, V. C., \& Di Sciascio, E. (2012, January). Movie Recommendation with DBpedia. In 3rd Italian Information Retrieval workshop (IIR) (pp. 101-112). [GS search]

Nash, S. S. (2005). Learning objects, learning object repositories, and learning theory: Preliminary best practices for online courses. Interdisciplinary Journal of Knowledge and Learning Objects, 1(2), 217-228. [GS search]

Navigli, R., \& Ponzetto, S. P. (2010, July). BabelNet: Building a very large multilingual semantic network. In Proceedings of the 48th annual meeting of the association for computational linguistics (pp. 216-225). [GS search]

Neves, D. E., Brandão, W. C. , \& Ishitani, L. (2016). Methodology for recommendation and aggregation of Learning Objects in SCORM. Brazilian Journal of Computers in Education, 24(01), 11. doi: 10.5753/rbie.2016.24.1.11. [GS search]

Oliveira, P., \& Rocha, J. (2013, April). Semantic annotation tools survey. In Computational Intelligence and Data Mining (CIDM), 2013 IEEE Symposium on (pp. 301-307). doi: 10.1109/CIDM.2013.6597251. [GS search]

Oren, E., Delbru, R., Catasta, M., Cyganiak, R., Stenzhorn, H., \& Tummarello, G. (2008). Sindice. com: a document-oriented lookup index for open linked data. International Journal of Metadata, Semantics and Ontologies, 3(1), 37-52. doi: 10.1504/IJMSO.2008.021204 
Qazi, A., \& Goudar, R. H. (2016). Emerging Trends in Reducing Semantic Gap towards Multimedia Access: A Comprehensive Survey. Indian Journal of Science and gy, 9(30). doi: 10.17485/ijst/2016/v9i30/99072

Raimond, Y., \& Lowis, C. (2012). Automated interlinking of speech radio archives. Linked Data on the Web (LDOW), 937. [GS search]

Ramalho, J. C. (2000). Anotação Estrutural de Documentos e sua Semântica, PhD Thesis, Departamento de Informática, Escola de Engenharia, Universidade do Minho, Braga, Portugal. [GS search]

Sack, H., \& Waitelonis, J. (2010, September). Exploratory semantic video search with yovisto. In Semantic Computing (ICSC), 2010 IEEE Fourth International Conference on (pp. 446447). doi: 10.1109/ICSC.2010.98. [GS search]

Shen, W., Wang, J., Luo, P., \& Wang, M. (2012, April). Linden: linking named entities with knowledge base via semantic knowledge. In Proceedings of the 21 st international conference on World Wide Web (pp. 449-458). doi: 10.1145/2187836.2187898. [GS search]

Shi, Y., Larson, M., \& Hanjalic, A. (2014). Collaborative filtering beyond the user-item matrix: A survey of the state of the art and future challenges. ACM Computing Surveys (CSUR), 47(1), 3. doi: 10.1145/2556270. [GS search]

Slimani, T. (2013). Semantic annotation: The mainstay of semantic web. In International Journal of Computer Applications Technology and Research, 2(6), 763 - 770. doi: 10.7753/IJCATR0206.1025. [GS search]

Vargas, S., \& Castells, P. (2011, October). Rank and relevance in novelty and diversity metrics for recommender systems. In Proceedings of the fifth ACM conference on Recommender systems (pp. 109-116). doi: 10.1145/2043932.2043955. [GS search]

Vinciarelli, A. (2005). Noisy text categorization. IEEE Transactions on Pattern Analysis and Machine Intelligence, 27(12), 1882-1895. doi: 10.1109/TPAMI.2005.248. [GS search]

Waitelonis, J., Sack, H., Hercher, J., \& Kramer, Z. (2010, April). Semantically enabled exploratory video search. In Proceedings of the 3rd international semantic search workshop (p. 8). doi: $\underline{10.1145 / 1863879.1863887}$

Yang, H., \& Meinel, C. (2014). Content based lecture video retrieval using speech and video text information. IEEE Transactions on Learning Technologies, 7(2), 142-154. doi: 10.1109/TLT.2014.2307305. [GS search]

Zhu, G., \& Iglesias, C. A. (2017). Computing Semantic Similarity of Concepts in Knowledge Graphs. IEEE Transactions on Knowledge and Data Engineering, 29(1), 72-85. doi: 10.1109/TKDE.2016.2610428. [GS search] 